\title{
Management of Early Glottic Cancer Treated by CO2 Laser According to Surgical-Margin Status: A Systematic Review of the Literature
}

\author{
Barbara Verro $^{1}$ Giuseppe Greco ${ }^{1}$ Enzo Chianetta ${ }^{1}$ Carmelo Saraniti ${ }^{1}{ }^{\circledR}$ \\ ${ }^{1}$ Department of Biomedicine, Neuroscience and Advanced \\ Diagnostics, ENT Clinic, University of Palermo, Palermo, Italy \\ Address for correspondence Carmelo Saraniti, MD, PhD, Dipartimento \\ di Biomedicina, Neuroscienze e Diagnostica Avanzata, Clinica \\ Otorinolaringoiatrica, Università degli Studi di Palermo, Via del Vespro, 129, \\ Int Arch Otorhinolaryngol 2021;25(2):e301-e308. \\ Palermo, 90127, Italy (e-mail: carmelo.saraniti@unipa.it).
}

\begin{abstract}
Introduction Transoral laser microsurgery (TLM) is the treatment of choice for Tis-T2 squamous cell glottic carcinomas due to its advantages compared with open surgery and radiotherapy. However, the $\mathrm{CO}_{2}$ laser beam causes changes and damage on the specimens, making the histological assessment of resection margins, the gold standard for confirming radical tumor resection, sometimes difficult.

Objective To assess the different ways to manage patients depending on the status of the histopathological margin according to recent studies to detect the most commonly shared therapeutic strategy.

Data Synthesis We analyzed the literature available on the PubMed and Web of Science databases, including only articles published since 2005, using specific keywords to retrieve articles whose titles and abstracts were read and analyzed independently by two authors to detect relevant studies. Therefore, we focused on disease-free survival, overall survival, local control, laryngeal preservation, and disease-specific survival. Thus, 17 studies were included in the present review; they were grouped according to the status of the histological margin, and we analyzed the different management policies described in them. This analysis showed that there is not a shared strategy, though in most studies the

Keywords

- glottic cancer

- squamous cell carcinoma

- laser surgery

- surgical margin

- second-look surgery authors performed a second-look surgery in the cases of positive margins and a close followup in cases of negative ones. The main disagreement is regarding the management of close or non-valuable resection margins, since some some authors performed a second-look surgery, and others, a close follow-up.

Conclusions Definitely, the most shared policy is the second-look surgery in case of positive surgical margins, and a close follow-up in case of close or non-valuable resection margins.
\end{abstract}

\section{Introduction}

Transoral laser microsurgery (TLM) was first used for the treatment of early glottic cancer in 1972, as described by Strong and Jako. ${ }^{1}$ To date, TLM is the treatment of choice for

received

April 21, 2020

accepted

May 10, 2020

published online

September 24, 2020
DOI https://doi.org/

10.1055/s-0040-1713922. ISSN 1809-9777.
Tis-T2 squamous cell glottic carcinomas. Indeed, this surgical procedure has many advantages compared with open surgery and radiotherapy (RT), such as: lower morbidity, shorter hospitalization, lower costs, and better organ preservation, with a lower impact on voice quality and swallowing, (c) 2020. Fundação Otorrinolaringologia. All rights reserved.

This is an open access article published by Thieme under the terms of the Creative Commons Attribution-NonDerivative-NonCommercial-License, permitting copying and reproduction so long as the original work is given appropriate credit. Contents may not be used for commercial purposes, or adapted, remixed, transformed or built upon. (https://creativecommons.org/ licenses/by-nc-nd/4.0/)

Thieme Revinter Publicações Ltda., Rua do Matoso 170, Rio de Janeiro, RJ, CEP 20270-135, Brazil 


\section{Key Points}

- To date, TLM is the treatment of choice for Tis-T2 squamous cell glottic carcinomas.

- The $\mathrm{CO}_{2}$ laser beam could impair the histological assessment of the resection margins, which is the gold standard to confirm radical tumor resection.

- Second-look TLM is the most performed strategy in case of positive surgical margins.

- Close follow-up is the most shared policy in case of close or non-valuable resection margins.

- In cases of negative resection margins, follow-up represents the best approach.

ensuring a radical excision of the lesion. ${ }^{2,3}$ Nevertheless, to date, the main critical questions involve the histological evaluation of the surgical resection margins and the management of the patients according to the status of the margins., ${ }^{4,5}$ In fact, some authors suggest a "wait and see" policy, 6,7 while others propose a second-look microlaryngoscopy $^{8,9}$ or RT when the resection margins are positive and/or close and/or unclear. The surgical margin is considered positive if the tumor is on the specimen's edge, and close if there is a close distance between the tumor and the margin itself that is not adequate to consider the excision completely safe. The main disagreement in the literature is regarding the margin-to-tumor distance. Indeed, some authors suggest $2 \mathrm{~mm}$ as a safe distance, ${ }^{7,10}$ while others consider $0.5 \mathrm{~mm},{ }^{6,11-13}$ and most suggest $1 \mathrm{~mm} .^{8,14-16}$ Moreover, the surgical margin is defined as non-valuable if laser artifacts such as carbonization make the assessment of the specimen's edge difficult. The resection margin is assessed as negative by a pathologist if it is free of tumor, with a proper tumor-to-margin distance, which differs from study to study.

The aim of the present review was to analyze the different ways to manage patients depending on the status of the histopathological margin according to recent studies to try to detect the most commonly shared therapeutic strategy. Therefore, we focused on disease-free survival (DFS), overall survival (OS), local control (LC), laryngeal preservation (LP), and disease-specific survival (DSS).

\section{Review of the Literature}

\section{Search Methodology}

We analyzed the literature available on the PubMed and Web of Science databases, including only articles published since 2005. The search strategy consisted of reading titles and abstracts independently by two authors (CS and BV) to detect relevant articles that would be studied in their entirety. We also searched for articles listed in the references of the pertinent studies. For the research, we used the following keywords: transoral laser microsurgery or transoral laser cordectomy or $\mathrm{CO} 2$ laser and early glottic cancer or glottic cancer and/or second-look and/or margin status or surgical margins. The inclusion criteria were early glottic cancer (Tis, T1, T2); TLM as the first therapeutic approach for glottic lesion; management according to the status of the surgical margin; absence of nodal and distant metastases (NOM0); patients without previous RT or surgery for laryngeal cancer; reporting of at least two of the following: DFS, OS, LC, LP, DSS; and mean or median follow-up period of at least 40 months. Studies were excluded if: they were reviews or editorials or opinions or case reports with fewer than twenty patients; articles published in languages other than English; studies that also included primary supraglottic or subglottic cancer; the patients who had a glottic lesion were first treated with CT and/or RT or endoscopic microsurgery.

\section{Study Selection}

- Fig. 1 shows the method of selection of the articles. In total, 17 studies were included in the present review, and their characteristics are shown in -Table $\mathbf{1}$ and -Table 2.

\section{Management of Positive Surgical Margin}

In cases of positive resection margin, a second-look surgery was the most followed approach. $8,17,18$ Other authors, such as Hendriksma et $\mathrm{al}^{14}{ }^{14}$ Hartl et $\mathrm{al}^{7}{ }^{7}$ and Lee $\mathrm{HS},{ }^{11}$ suggest a second-look TLM or close follow-up according to the surgeon's evaluation during surgery or in cases of suspicion of relapse during the follow-up.

On the other hand, Fiz et al $^{19}$ usually followed a different policy according to the number and depth of the positive margins: they prefer the close follow-up only if a superficial margin is positive, and the second-look procedure, open surgery or RT strategies if more than one superficial margin or the deep margins are positive. By using this strategy, Fiz et al achieved a 5-year DFS in $77.2 \%$, DSS in $98.3 \%$, and LP in $96.2 \%$ of their sample. The same protocol was followed by Galli et $\mathrm{al}^{20}$ and Lucioni et al. ${ }^{21}$

Moreover, in another study, Lucioni et $\mathrm{al}^{15}$ suggested performing the second-look surgery or RT in case of positive deep surgical margins, and close follow-up for positive superficial margins, achieving a 3-year DFS in $84,7 \%$, a 3 -year DSS in $97,8 \%$ and a 5 -year OS in $91,4 \%$ of their sample.

On the contrary, Ansarin et $\mathrm{al}^{16}$ perform a second look with TLM only if a resection margin is positive, and RT if there is more than 1 positive margin, achieving a 5-year OS in $90.01 \%$, LP in $97.1 \%$, and a 8 -year DFS in $88.2 \%$ of their sample.

However, other authors $6,10,12,13,22$ always perform a close follow-up. Thus, in 2015, Hoffmann et al $^{12}$ achieved a 5-year OS in $79.2 \%$, DSS in $91.5 \%$, DFS in $61.7 \%$, LP in $93.4 \%$, and LC in $74.4 \%$ of their sample. In their case series, Charbonnier et al ${ }^{10}$ had similar findings, with a 5 -year OS in $88 \%$, DFS in $73 \%$, and LC in $79 \%$ of their sample.

Preuss et $\mathrm{al}^{23}$ performed second- and third-look TLMs in all patients regardless of the status of the resection margin, with a 5 -year OS in $100 \%$, and a 5 -year DFS in $96.3 \%$ of their sample. 


\section{PRISMA 2009 Flow Diagram}

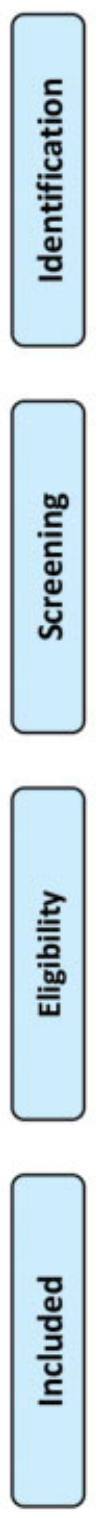

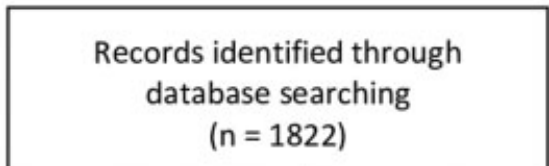

$(\mathrm{n}=1822)$
Additional records identified through other sources $(n=3)$

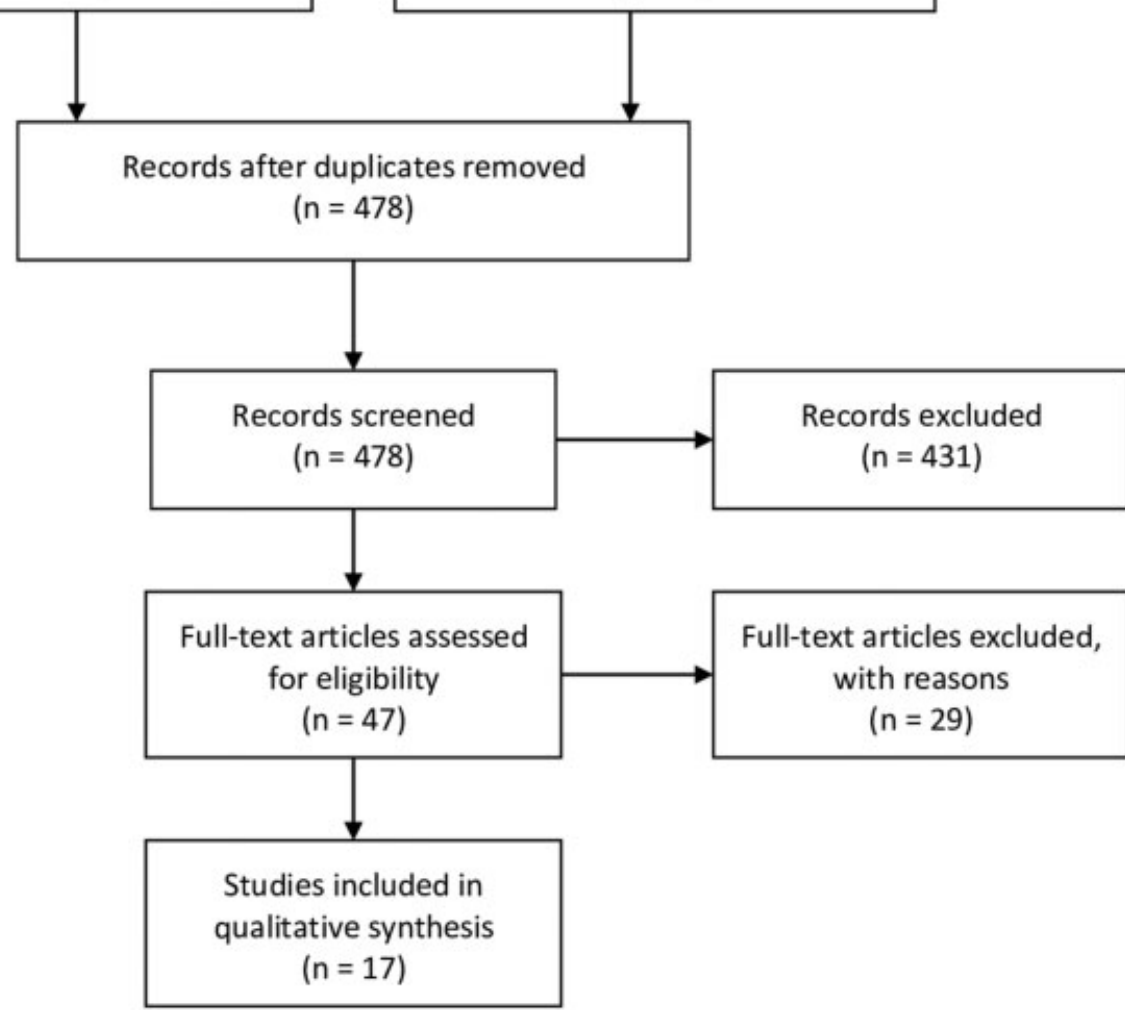

From: Moher D, Liberati A, Tetzlaff J, Altman DG, The PRISMA Group (2009). Preferred Reporting ftems for Systematic Reviews and MetaAnalyses: The PRISMA Statement. PLoS Med 6(7): e1000097. doi:10.1371/journal.pmed1000097

For more information, visit www.prisma-statement.org.

Fig. 1 Preferred Reporting Items for Systematic Reviews and Meta-Analyses (PRISMA) 2009 flow diagram of the selection process of studies in the current literature. 


\begin{tabular}{|c|c|c|c|c|c|c|c|c|c|c|c|c|c|c|c|c|c|}
\hline & 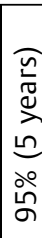 & - & 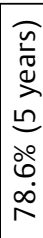 & 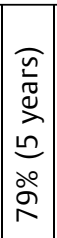 & 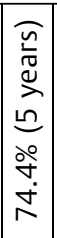 & - & - & 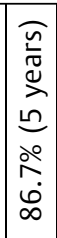 & 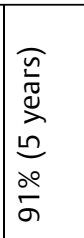 & 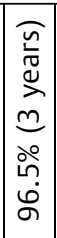 & - & - & - & 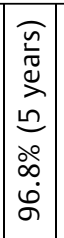 & - & 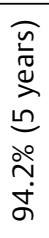 & 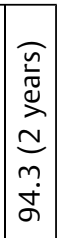 \\
\hline 9 & 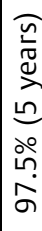 & - & 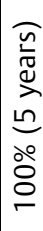 & - & 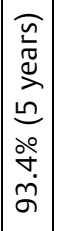 & 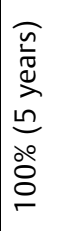 & 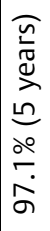 & 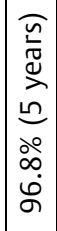 & - & 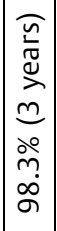 & 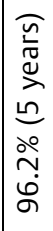 & - & - & 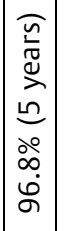 & 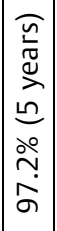 & 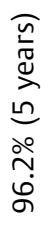 & 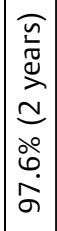 \\
\hline 虫 & 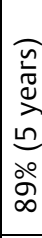 & 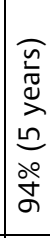 & - & 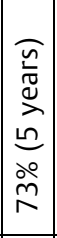 & 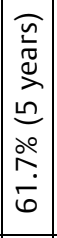 & 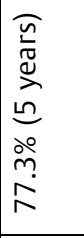 & 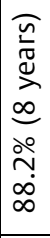 & 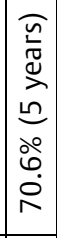 & 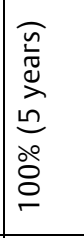 & - & 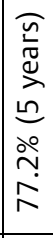 & 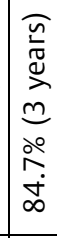 & 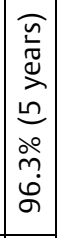 & - & 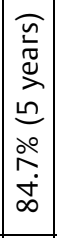 & 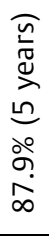 & - \\
\hline a & 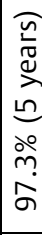 & - & 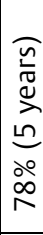 & - & 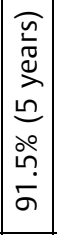 & - & - & 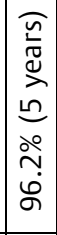 & - & 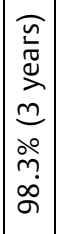 & 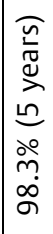 & 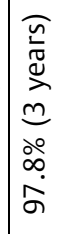 & - & 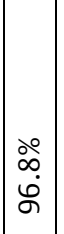 & 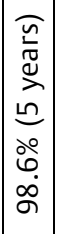 & 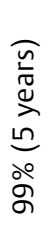 & 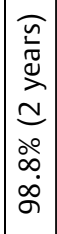 \\
\hline ○ & - & 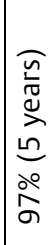 & 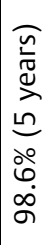 & 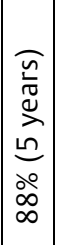 & 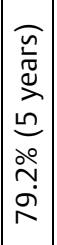 & 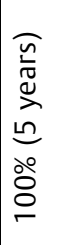 & 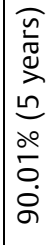 & 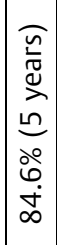 & 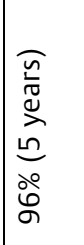 & 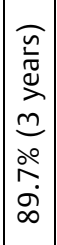 & - & 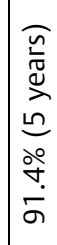 & 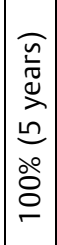 & - & & 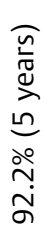 & 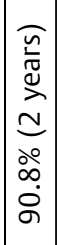 \\
\hline 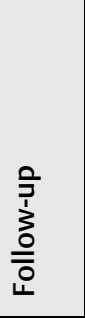 & 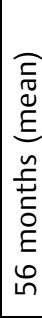 & 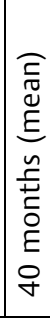 & 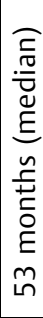 & 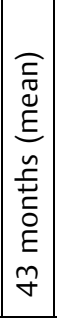 & 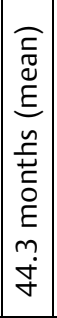 & 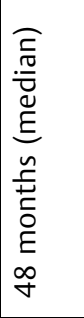 & 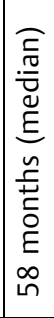 & 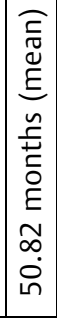 & 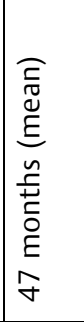 & 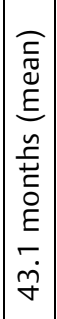 & 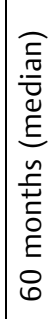 & 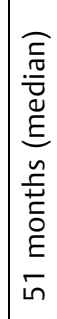 & 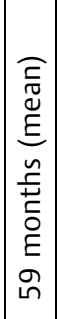 & 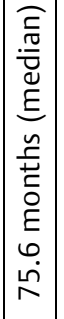 & 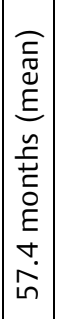 & 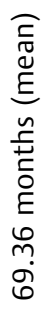 & 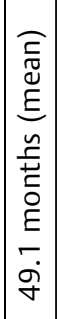 \\
\hline 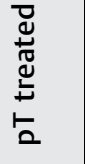 & $\begin{array}{l}F_{\dot{n}} \\
\stackrel{\ddots}{F}\end{array}$ & $\stackrel{\sigma}{F}$ & 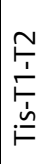 & $\frac{F}{F}$ & $\mid \begin{array}{l}\stackrel{N}{\dot{I}} \\
\dot{F} \\
\dot{F}\end{array}$ & $\frac{F}{F}$ & $\begin{array}{l}\stackrel{N}{i} \\
\stackrel{i}{i} \\
\stackrel{\tilde{v}}{F}\end{array}$ & 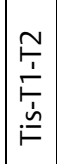 & $\begin{array}{l}\stackrel{F}{i} \\
F \vec{F} \\
i \underline{E}\end{array}$ & $\begin{array}{l}F \\
F \\
F\end{array}$ & 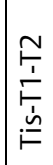 & $\frac{F}{F}$ & $\begin{array}{l}F \\
F \\
F\end{array}$ & 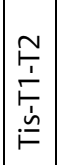 & $\frac{F}{F}$ & 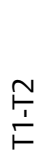 & $\begin{array}{l}F \\
F\end{array}$ \\
\hline 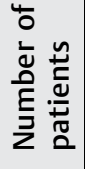 & $\stackrel{9}{1}$ & ర্ & ১ & $\mp$ & Һ & $\stackrel{0}{-}$ & $\bar{m}$ & $\bar{\sim}$ & 望 & $\stackrel{\infty}{\infty}$ & $\hat{\sigma}$ & $\stackrel{\infty}{\sim}$ & $\stackrel{\infty}{\infty}$ & $n$ & $\mathbb{N}$ & $\stackrel{\infty}{=}$ & $\stackrel{ }{\Sigma}$ \\
\hline 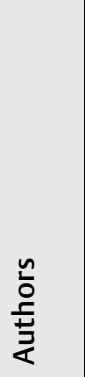 & 年 & 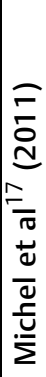 & 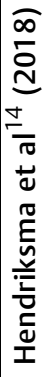 & 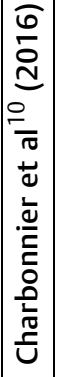 & 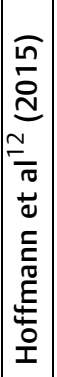 & 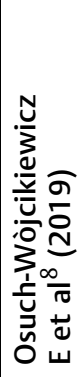 & 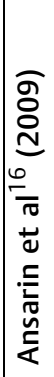 & 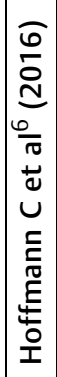 & 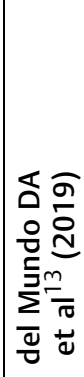 & 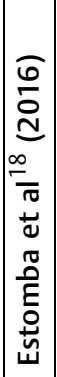 & 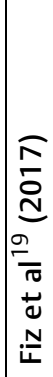 & 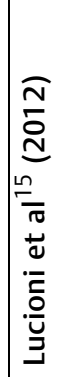 & 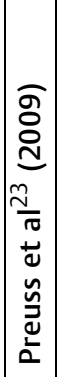 & 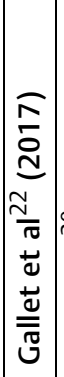 & 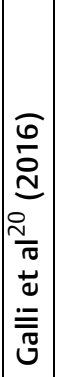 & 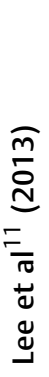 & 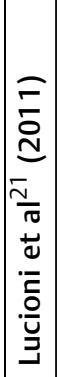 \\
\hline
\end{tabular}




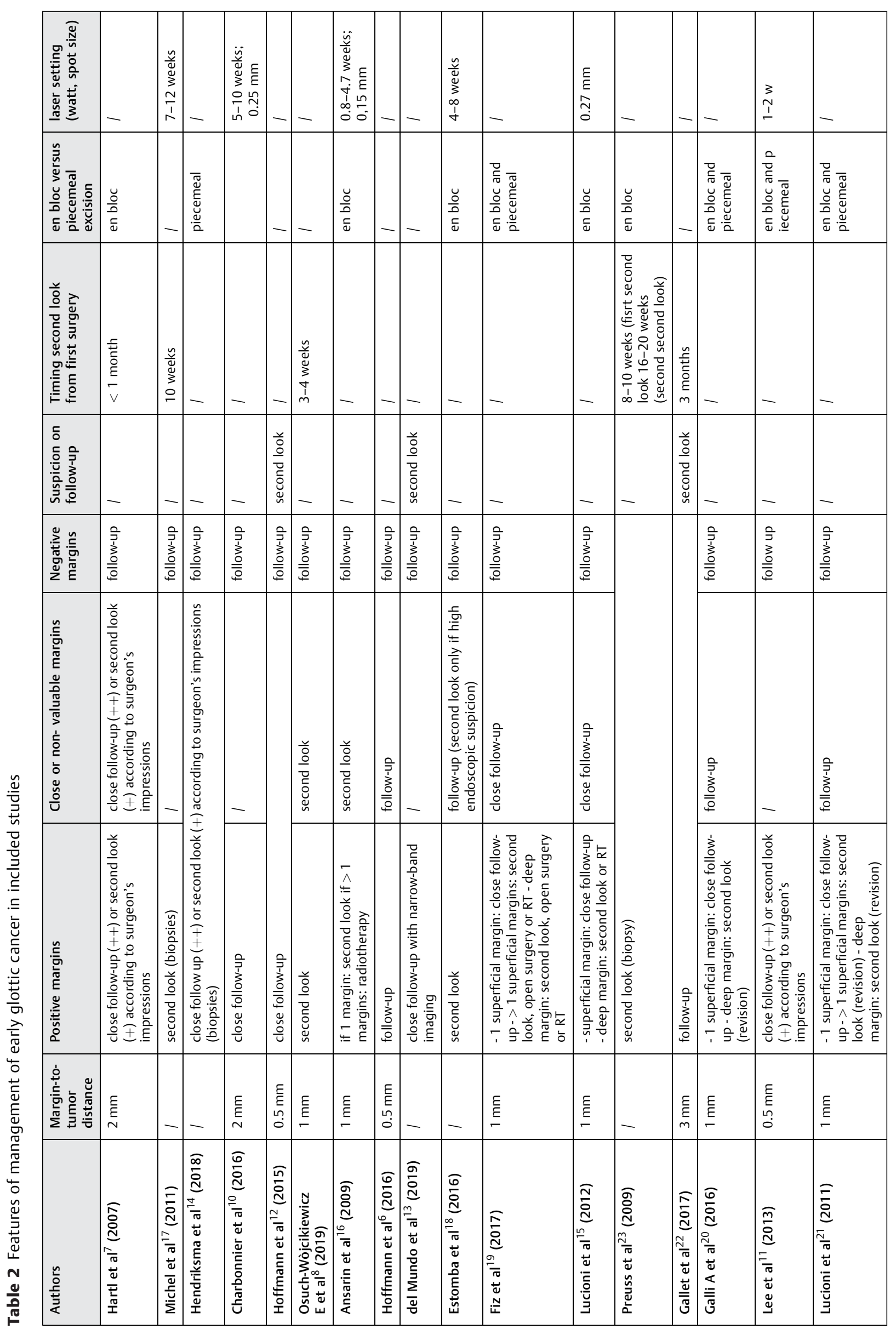


Management of Close or Non-valuable Surgical Margins Regarding close or non-valuable resection margins, in the literature the most followed strategy was the close follow-up. $6,11,12,15,18,19$ However, Hendriksma et al ${ }^{14}$ and Hartl et $\mathrm{al}^{7}$ decided the most proper strategy between close follow-up and second-look surgery on a case-by-case basis. According to these authors, a surgeon's intraoperative evaluation is just as important as the histological assessment. Moreover, Hartl et $\mathrm{al}^{7}$ argue that in cases of disagreement between the surgeon and the pathologist (regarding positive or non-valuable margins), they usually performed a secondlook TLM. In the literature, on the contrary, other authors recommend the second-look approach as a general rule if the margins are close or non-valuable. ${ }^{8,15,23}$

Some studies did not examine this kind of surgical-margin status, assessing only positive and negative ones. ${ }^{10,12,13}$

\section{Management of Negative Surgical Margins}

In the literature, there is no disagreement concerning the most proper approach in cases of negative resection margins. Thus, all authors suggest the follow-up. Only Preuss et $\mathrm{al}^{23}$ performed at least two second-look microlaryngoscopies in cases of negative surgical margins.

\section{Discussion}

\section{Summary of the Main Results}

The present systematic review analyzed the different ways to manage patients previously treated with TLM depending on the histopathological status of the margin according to recent studies to try to detect the most commonly shared therapeutic strategy. The analysis showed that there is not a shared strategy, though most authors performed a second-look surgery in cases of positive margins, and follow-up in cases of negative ones. Regarding the management of close or nonvaluable resection margins, there is a disagreement, since some authors performed a second-look, and others, close follow-up.

\section{Challenges in the Histological Assessment of Resection Margins}

The $\mathrm{CO}_{2}$ laser beam causes changes and damage on the specimens, making the histological assessment of the resection margins, which is the gold standard to confirm radical tumor resection, sometimes difficult. In particular, laserinduced changes and damages could be due to the laser setting (spot size and power beam), which is adjusted by the surgeon, and the TLM experience of the surgeon themselves. Moreover, other issues that could impair margin assessment are small size and/or shrinking of the specimen during the histological preparation, carbonization, and difficulty in freezing sections. ${ }^{4,11,14,24-28}$ In particular, in the case of small specimens, making frozen sections or correctly orienting them is usually not possible, especially if the lesion is excised with the piece-meal technique. Indeed, in the literature, most authors ${ }^{7,15,16,18,23}$ report they prefer the en-bloc technique to ensure an adequate histopathological assessment. Some authors ${ }^{11,19-21}$ report using the en-bloc or piece-meal technique depending on the location of the tumor, the depth of the infiltration, the laryngeal exposure, and the size of the tumor. In particular, Lee et $\mathrm{al}^{11}$ argued that the en-bloc resection is recommended for small superficial tumors, while the piece-meal resection enables the assessment of the depth of the infiltration, especially in cases of extensive tumors. Only Hendriksma et a ${ }^{14}$ routinely prefer the multi-bloc technique. Their standard approach consists of resection of the tumor in two pieces and another five biopsies around the tumor bed.

Furthermore, the $\mathrm{CO}_{2}$ laser induces an area of carbonization of $0.3 \mathrm{~mm}$ around the section line, which should be considered in the evaluation of margin status. ${ }^{26}$ Indeed, since this carbonization makes it difficult to differentiate between positive and unclear resection margins, some authors ${ }^{11,14}$ decided to group these two different margins together, and recommend the same treatment approach, which is secondlook or close follow-up according to the surgeon's evaluation. Preuss et $\mathrm{al}^{23}$ routinely performed at least two second looks in all patients regardless of the status of the surgical margin due to charring artifacts that would not enable tumor detection in resection margins.

In their study, Buchanan et $\mathrm{al}^{29}$ pointed out that the $\mathrm{CO}_{2}$ laser induces a thermal cytologic damage that could result in misinterpretation or difficult assessment of margin status. In particular, they report that the $\mathrm{CO}_{2}$ laser at a higher power causes less tissue damage thanks to the greater speed of the beam and, consequently, reduced contact between it and the cell. Furthermore, a small size of the laser beam spot $(0.25 \mathrm{~mm})$ ensures greater accuracy in the surgery and reduces tissue carbonization. However, in the literature, the $\mathrm{CO}_{2}$ laser setting differed from study to study. ${ }^{10,11,15-18}$ Charbonnier et $\mathrm{al}^{10}$ performed TLM with a laser beam power between $5 \mathrm{~W}$ and $10 \mathrm{~W}$, and a beam spot of $0.25 \mathrm{~mm}$, while Ansarin et $\mathrm{al}^{16}$ preferred between $0.8 \mathrm{~W}$ and $4.7 \mathrm{~W}$ of laser power, and a spot size of $0.15 \mathrm{~mm}$. Charbonnier et $\mathrm{al}^{10}$ treated 110 patients with 30 cases of positive resection margins; during the follow-up, 23 patients had recurrence, and among these, only 10 cases had positive margins, supporting the findings of Buchanan et al. ${ }^{29}$ Meanwhile, Lee et a ${ }^{11}$ performed TLM with a power setting of $1 \mathrm{~W}$ to $2 \mathrm{~W}$ in 118 patients with 65 cases of negative margins and 43 cases of positive margins: only 14 patients had recurrence during the follow-up, suggesting that the low laser power setting did not impair the correct histopathological assessment of the resection margins.

\section{Photocoagulation of the Surgical Bed}

Laser photocoagulation (LPC) of the surgical bed can be considered an effective technique to destroy any tumor remnant, thus reducing the risk of recurrence. ${ }^{10,15,18}$ Lucioni et $\mathrm{al}^{15}$ vaporized tissue around resection margins with the following laser setting: circular spot shape, spot size of $1.6 \mathrm{~mm}$, and beam power of $16 \mathrm{~W}$. They achieved a low local recurrence rate in cases of non-valuable, close and positive superficial margins. However, they also demonstrated that LPC is not able to destroy tumor cells in cases of positive deep margins. Furthermore, LPC does not impair voice quality, and so it can be routinely performed. 


\section{Margin-to-tumor Distance}

Another main issue regarding TLM in glottic cancer concerns the margin-to-tumor distance. In the literature, this parameter differs from study to study. In particular, most authors $8,15,16,19-21$ establish $1 \mathrm{~mm}$ as a safe distance, while some $^{6,11,12}$ suggest $0.5 \mathrm{~mm}$, and a few ${ }^{7,10}$ report $2 \mathrm{~mm}$. Moreover, the laser beam has a different effect on carcinoma and on healthy tissue that could help detect the tumor borderline. As a result, as aforementioned, the experience of the surgeon with the laser is very important for a radical tumor excision.

\section{Management Policies According to the Status of the Resection Margins}

Difficult histopathological assessment of resection margins, as well as disagreement over the margin-to-tumor distance result in different management policies in cases of positive, close and negative resection margins. Indeed, in cases of positive and close resection margins, some adopt a "wait and see" strategy with endoscopic follow-up, while others suggest a second-look TLM. In particular, in cases of positive margins, the literature reports that second-look TLM is the most accepted practice, with some differences from author to author. Some, such as Fiz et al, ${ }^{19}$ Galli et al, ${ }^{20}$ and Lucioni et $\mathrm{al}^{21}$ perform close follow-up in cases of just one positive superficial margin, and the second-look procedure in cases of more than one positive superficial margin or deep margins. Doing so, Fiz et $\mathrm{al}^{19}$ achieved a 5-year DFS in $77.2 \%$, DSS in $98.3 \%$, and LP in $96.2 \%$ of their sample. Other authors, such as Charbonnier et $\mathrm{al}^{10}$ and Hoffmann et $\mathrm{al}^{12}$ prefer a close follow-up approach in cases of positive margins, regardless of the depth of the infiltration and the number of positive margins. Thus, Hoffmann achieved a 5-year DFS, DSS, LP, OS and LC in $61.7 \%, 91.5 \%, 93.4 \%$, $79.2 \%$, and $74.4 \%$ of their sample respectively. ${ }^{12}$ By comparing the results of these different strategies regarding the 5year DFS, DSS and LP, the performance of the second-look surgery has better results than the close follow-up in cases of positive resection margins, ensuring a lower risk of recurrence and of invasive surgery, which can impair organ preservation. Preuss et $\mathrm{al}^{23}$ suggest performing second- and third-look TLM in all patients regardless of the status of the resection margins, with a 5-year DFS in $96.3 \%$, and a 5-year OS in $100 \%$ of their sample. Indeed, in their study, they found an increased incidence of residual tumor with two successive second-look TLMs, which enabled them to achieve complete resection of the tumor and early detection of recurrence, as demonstrated by a 5-year DFS in $96.3 \%$ of their sample, compared with a 5-year DFS in 73\% and 61,7\% in the studies by Charbonnier ${ }^{10}$ and Hoffmann ${ }^{12}$ respectively.

Regarding close or non-valuable margins, close follow-up represents the most widely accepted approach. $6,11,12,15,17,19$ However, some surgeons perform follow-up or second-look TLM in a case-by-case basis. ${ }^{7,14}$ Actually, they have stated the importance of the surgeon's intraoperative evaluation over the histopathological assessment. Thus, in cases of a histological evaluation of positive or close resection margins, the surgeon performs a second-look surgery or follow-up according to his/her evaluation of the radicalness of the of surgery.

Moreover, in 2014, after reviewing the then current literature, the European Laryngological Society (ELS) issued some evidence-based recommendations; in particular, it established that second-look TLM is mandatory in cases of positive margins, and recommended if the margins are close or non-valuable. ${ }^{30}$

In cases of negative surgical margins, all authors suggest follow-up, except Preuss et al, ${ }^{23}$ as aforementioned. However, several studies ${ }^{7,13,19,22}$ have reported the possibility of local recurrence even in cases of negative resection margins. Based on this point of view, follow-up, preferably with narrow-band imaging (NBI), is important to detect early recurrence. ${ }^{13,18}$ In fact, NBI is an endoscopic system that enables a better evaluation of the microvascular pattern in cases of preneoplastic and neoplastic lesions of the mucosa that cannot be examined with white-light endoscopy. ${ }^{31}$ So, NBI enables the detection of the early recurrence of the tumor and its superficial extension, but cannot evaluate the depth of the infiltration.

\section{Timing of Second-Look TLM}

In order to perform the early detection of recurrence or a residual tumor, rather than close follow-up, Preuss et $\mathrm{al}^{23}$ prefer performing second-look TLM routinely. Moreover, they argue the importance of a short interval between the first and second TLMs. In fact, according to their protocol, the patients undergo two second-look procedures, at 8 and 16 weeks after the first surgery, regardless of the status of the resection margins, since they often found tumor remnants only after the second look. However, in the literature, there is discordance regarding the timing of the second-look TLM. Some authors ${ }^{7,8}$ recommend performing the second surgery $\sim 3$ to 4 weeks after the first TLM, while Gallet et al $^{22}$ recommend it 3 months after. The latter argue that this period is adequate to detect any residual tumors and to avoid overtreatment at the same time, because, based on their experience, they found that recurrence usually occurs several months after surgery. The laser effects in terms of thermal damage and carbonization on treated tissue should also be considered and, therefore, the second-look surgery should be performed a few months after the first surgery, which is a sufficiently long time to ensure tissue healing and, thus, a radical tumor resection. However, in the literature, the timing of the second look is not usually reported, likely because the majority of clinicians do not consider it decisive for the outcome of the final surgery, which is complete tumor resection. The ELS does not make any recommendations regarding this, but it states that the timing of the secondlook procedure is still controversial, and that it ranges from 1 to 8 months after the first surgery. ${ }^{30}$

\section{Final Comments}

The objective of the present review was to detect a common policy in the treatment and follow-up of early glottic cancer after the first TLM surgery. We analyzed the different strategies 
present in literature: second-look TLM or follow-up according to the different statuses of the resection margins. In particular, in the literature, the most accepted strategy is second-look TLM in case of positive surgical margins, and close follow-up in case of close or non-valuable ones. Furthemore, regarding negative resection margins, most authors suggest follow-up.

However, due to several issues that impact on the choice of policy, further studies, specially about laser-induced changes on resection margins, would be needed to establish a shared guideline.

\section{Conflict of Interests}

The authors have no conflict of interests to declare.

\section{References}

1 Strong MS, Jako GJ. Laser surgery in the larynx. Early clinical experience with continuous CO 2 laser. Ann Otol Rhinol Laryngol 1972;81(06):791-798

2 Grant DG, Salassa JR, Hinni ML, Pearson BW, Hayden RE, Perry WC. Transoral laser microsurgery for untreated glottic carcinoma. Otolaryngol Head Neck Surg 2007;137(03):482-486

3 Preuss SF, Cramer K, Klussmann JP, Eckel HE, Guntinas-Lichius O. Transoral laser surgery for laryngeal cancer: outcome, complications and prognostic factors in 275 patients. Eur J Surg Oncol 2009;35(03):235-240

4 Sigston E, de Mones E, Babin E, et al. Early-stage glottic cancer: oncological results and margins in laser cordectomy. Arch Otolaryngol Head Neck Surg 2006;132(02):147-152

5 Fakhry N, Vergez S, Babin E, et al. Management of surgical margins after endoscopic laser surgery for early glottic cancers: a multicentric evaluation in French-speaking European countries. Eur Arch Otorhinolaryngol 2015;272(06):1465-1469

6 Hoffmann C, Hans S, Sadoughi B, Brasnu D. Identifying outcome predictors of transoral laser cordectomy for early glottic cancer. Head Neck 2016;38(Suppl 1):E406-E411

7 Hartl DM, de Monès E, Hans S, Janot F, Brasnu D. Treatment of early-stage glottic cancer by transoral laser resection. Ann Otol Rhinol Laryngol 2007;116(11):832-836

8 Osuch-Wòjcikiewicz E, Rzepakowska A, Sobol M, Bruzgielewicz A, Niemczyk K. Oncological Outcomes of Co2 Laser Cordectomies for Glottic Squamous Cell Carcinoma With Respect to Anterior Commissure Involvement and Margin Status. Wiley Periodicals, 2019: $1-8$

9 Peretti G, Piazza C, Bolzoni A, et al. Analysis of recurrences in 322 Tis, T1, or T2 glottic carcinomas treated by carbon dioxide laser. Ann Otol Rhinol Laryngol 2004;113(11):853-858

10 Charbonnier Q Thisse AS, Sleghem L, et al. Oncologic outcomes of patients with positive margins after laser cordectomy for T1 and T2 glottic squamous cell carcinoma. Head Neck 2016;38(12): 1804-1809

11 Lee HS, Chun BG, Kim SW, et al. Transoral laser microsurgery for early glottic cancer as one-stage single-modality therapy. Laryngoscope 2013;123(11):2670-2674

12 Hoffmann C, Hans S, Sadoughi B, Brasnu D. Identifyngoutcomepredictors of transoral laser cordectomy for early glottic cancer. Laryngoscope $2015 ; \cdots \cdot \cdots: 1-6$

13 del Mundo DAA, Morimoto K, Masuda K, et al. Oncological and functionaloutcomes of transoral CO2 laser cordectomy for early glottic cancer. Auris Nasas Larynx 2019

14 Hendriksma M, Montagne MW, Langeveld TPM, Veselic M, van Benthem PPG, Sjögren EV. Evaluation of surgical margin status in patients with early glottic cancer (Tis-T2) treated with transoral
$\mathrm{CO}_{2}$ laser microsurgery, on local control. Eur Arch Otorhinolaryngol 2018;275(09):2333-2340

15 Lucioni M, Bertolin A, D’Ascanio L, Rizzotto G. Margin photocoagulation in laser surgery for early glottic cancer: impact on disease local control. Otolaryngol Head Neck Surg 2012;146(04):600-605

16 Ansarin M, Santoro L, Cattaneo A, et al. Laser surgery for early glottic cancer: impact of margin status on local control and organ preservation. Arch Otolaryngol Head Neck Surg 2009;135(04): 385-390

17 Michel J, Fakhry N, Duflo S, et al. Prognostic value of the status of resection margins after endoscopic laser cordectomy for T1a glottic carcinoma. Eur Ann Otorhinolaryngol Head Neck Dis 2011;128(06):297-300

18 Estomba CMC, Reinoso FAB, Velasquez AO, et al. Transoral CO2 laser microsurgery outcomes for early glottic carcinomas T1-T2. Int Arch Otorhinolaryngol 2016;20(03):212-217

19 Fiz I, Mazzola F, Fiz F, et al. Impact of Close and Positive Margins in Transoral Laser Microsurgery for Tis-T2 Glottic Cancer. Front Oncol 2017;7:245

20 Galli A, Giordano L, Sarandria D, Di Santo D, Bussi M. Oncological and complication assessment of $\mathrm{CO} 2$ laser-assisted endoscopic surgery for T1-T2 glottic tumours: clinical experience. Acta Otorhinolaryngol Ital 2016;36(03):167-173

21 Lucioni M, Marioni G, Bertolin A, Giacomelli L, Rizzotto G. Glottic laser surgery: outcomes according to 2007 ELS classification. Eur Arch Otorhinolaryngol 2011;268(12):1771-1778

22 Gallet P, Rumeau C, Nguyen DT, Teixeira PA, Baumann C, Toussaint B. "Watchful observation" follow-up scheme after endoscopic $\mathrm{CO}_{2}$ laser treatment for small glottic carcinomas: A retrospective study of 93 cases. Clin Otolaryngol 2017;42(06):1193-1199

23 Preuss SF, Cramer K, Drebber U, Klussmann JP, Eckel HE, GuntinasLichius O. Second-look microlaryngoscopy to detect residual carcinoma in patients after laser surgery for $\mathrm{T} 1$ and $\mathrm{T} 2$ laryngeal cancer. Acta Otolaryngol 2009;129(08):881-885

24 Saraniti C, Speciale R, Gallina S, Salvago P. Prognostic role of resection margin in open oncologic laryngeal surgery: survival analysis of a cohort of 139 patients affected by squamous cell carcinoma. Rev Bras Otorrinolaringol (Engl Ed) 2019;85(05): 603-610

25 Peretti G, Nicolai P, Redaelli De Zinis LO, et al. Endoscopic CO2 laser excision for Tis, T1, and T2 glottic carcinomas: cure rate and prognostic factors. Otolaryngol Head Neck Surg 2000;123(1 Pt 1):124-131

26 Brøndbo K, Fridrich K, Boysen M. Laser surgery of T1a glottic carcinomas; significance of resection margins. Eur Arch Otorhinolaryngol 2007;264(06):627-630

27 Dispenza F, Saraniti C, Mathur N, Dispenza C. Modified frontolateral laryngectomy in treatment of glottic T1(a-b) cancer with anterior commissure involvement. Auris Nasus Larynx 2010;37 (04):469-473

28 Shenoy AM, Prashanth V, Shivakumar T, et al. The utility of second look microlaryngoscopy after trans oral laser resection of laryngeal cancer. Indian J Otolaryngol Head Neck Surg 2012;64(02): 137-141

29 Buchanan MA, Coleman HG, Daley J, et al. Relationship between CO2 laser-induced artifact and glottic cancer surgical margins at variable power doses. Head Neck 2016;38(Suppl 1):E712-E716

30 Simo R, Bradley P, Chevalier D, et al. European Laryngological Society: ELS recommendations for the follow-up of patients treated for laryngeal cancer. Eur Arch Otorhinolaryngol 2014; 271(09):2469-2479

31 Campo F, D’Aguanno V, Greco A, Ralli M, de Vincentiis M. The prognostic value of addingNarrow-Band Imaging in transoral laser microsurgery for early glottic cancer: a review. Lasers Surg Med 2019; $\cdots \bullet: 1-6$ 